\title{
Difficulties in the Diagnosis of Myocarditis -Case report
}

\section{R. H. A. I. Rathnaweera ${ }^{1}$}

\begin{abstract}
Myocarditis is an elusive illness to study, diagnose and treat because the clinical presentation may range from nearly asymptomatic to overt heart failure. A 61 years old female developed fever with chills and rigors during a pilgrimage. After returning, she developed shortness of breath, with retrosternal chest pain and admitted to Teaching Hospital Karapitiya. An E.C.G. (Echocardiography) showed ST elevation in V1 - V6 and ST depression in II, III and AVF leads. The patient was treated as having an acute anterior ST elevation MI (myocardial infarction) treated with heparin and anti anginal drugs. The patient died about six hours after the admission. At the post-mortem, heart appeared flabby with $30 \%$ occlusion of the left coronary artery. Histopathology examination revealed acute myocarditis. Many secondary causes can lead to inflammation of the myocardium and therefore the diagnosis of myocarditis cannot be made by evidence of inflammation of the myocardium alone. Therefore, unless multiple, florid foci are found with myofibril necrosis, bland mononuclear foci that are purely interstitial and not involving muscle fibers must be disregarded.
\end{abstract}

Keywords: myocarditis, post-mortem histopathology

\section{INTRODUCTION}

Myocarditis is an elusive illness to study, diagnose and treat because the clinical presentation may range from nearly asymptomatic to overt heart failure and sudden death (1). It may even resemble acute coronary ischemic syndrome. Sometimes misdiagnosis of myocarditis is made and sometimes we mis the diagnosis of myocarditis.

\section{CASE REPORT}

Sixty one years old female, married, mother of six children, developed fever with chills and rigors for two days during a trip to Rathnapura. After returning home, on the following day morning, she developed shortness of breathing, together with retrosternal chest pain. No fever was present at that time. She was admitted to the Teaching Hospital Karapitiya, immediately and was taken to the E.T.U.
At the E. T. U. an urgent E.C.G. was taken and it showed ST elevation in V1 - V6 together with ST depression in II, III, and AVF leads. She was treated as for anterior ST elevation myocardial infarction, with heparin and anti anginal drugs. But she deteriorated gradually and died about six hours after admission to the E.T.U.

At the post-mortem, heart appeared flabby, with $30 \%$ occlusion of the anterior descending branch of the left coronary artery. All the other organs appeared unremarkable. Histological examination revealed diffuse mixed inflammatory cell infiltrates rich in lymphocytes. There was focal necrosis of myocardium around which inflammatory infiltrate was prominent (Figure 1 and 2). The above features are compatible with acute myocarditis, most probably viral in origin. 


\section{DISCUSSION}

Myocarditis refers to a process of diffuse inflammatory infiltrate and focal areas of necrosis of the myocardium. Under this category, inflammatory processes of the myocardium that result in injury to cardiac myocytes are grouped (2). However, the presence of inflammation alone is not diagnostic of myocarditis, because inflammatory infiltrate may also be seen as a secondary response in conditions such as ischemic injury (2). In myocarditis, by contrast, the inflammatory process is the cause of rather than a response to myocardial injury (2).

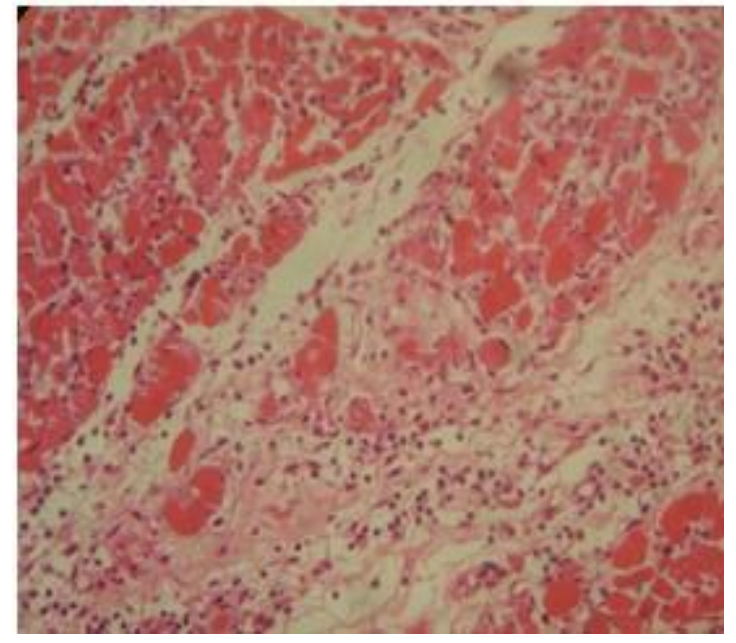

Figure 1: Lymphocyte rich inflammatory cell infiltrate with focal myocardial necrosis-lower (x10) magnification

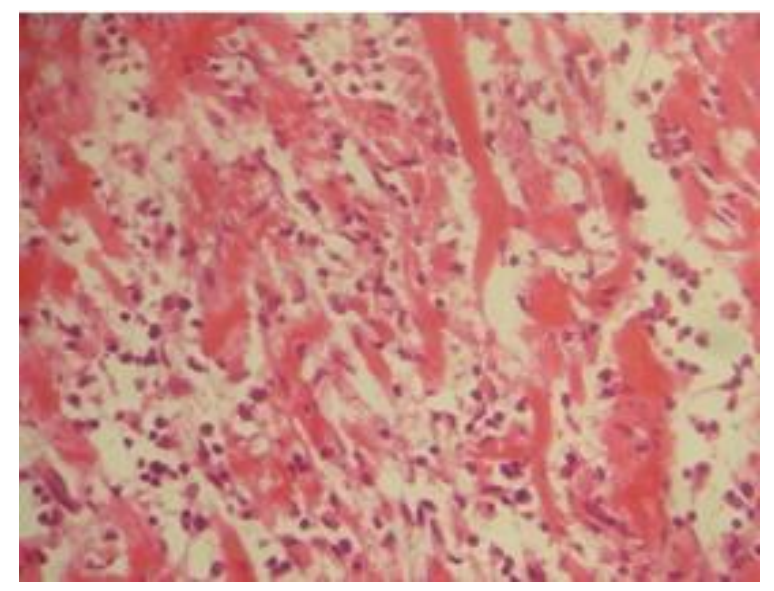

Figure 2: Lymphocyte rich inflammatory cell infiltrate with focal myocardial necrosis-higher (x40) magnification
Infections and particularly viruses are the most common cause of myocarditis. Coxackie viruses $\mathrm{A}$ and $\mathrm{B}$ and other entero viruses account for most of the myocarditis cases. Viral myocarditis, as in this case, is characterized by mononuclear inflammatory infiltrate composed largely of lymphocytes and associated myocyte injury (2). Other less common etiologic agents include Chlamydia, Rickettsia, Bacteria, Fungi and Protozoa. A morphologically distinctive form of myocarditis of uncertain cause, called giant cell myocarditis, is characterized by a wide spread inflammatory cellular infiltrate containing multinuclear giant cells interspersed with lymphocytes, eosinophils, plasma cells and macrophages and having at least focal but frequently extensive necrosis. These giant cells are either of macrophage or myocyte in origin. These variant cells carry a poor prognosis (2). Hypersensitivity myocarditis is characterized by an interstitial inflammatory infiltrate composed largely of eosinophils and mononuclear inflammatory cells predominantly localized to perivascular and large interstitial spaces. This form of myocarditis is associated with drug hypersensitivity.

The Dallas criteria were proposed in 1986 and provided a histopathological categorization by which the diagnosis of myocarditis could be established (3). According to the Dallas criteria myocarditis requires an inflammatory infiltrate and associated myocyte necrosis or damage not characteristic of an ischemic event. Borderline myocarditis requires a less intense inflammatory infiltrate and no light microscopic evidence of myocyte destruction (4). 
These criteria have been used exclusively by American investigators over the last two decades. Sampling error, variation in expert interpretation, variance with other markers of viral infection and immune activation in the heart and variance with treatment outcomes all suggest that the Dallas criteria are no longer adequate. It was demonstrated by biopsies done on post-mortem hearts of patients who had died with myocarditis that, from a single endomyocardial biopsy, histological myocarditis could be demonstrated in only $25 \%$ of samples (5). With more than 5 biopsies, Dallas criteria myocarditis could be diagnosed in approximately two thirds of subjects. This point outs the importance of taking multiple sections of the heart in any complete examination of the heart for heart disease.

\section{REFERENCES}

1. Payen-James J, Busuttil A. Forensic Medicine: Clinical and pathological aspects. $1^{\text {st }}$ ed. UK: Bath press 1td, 2003: 282-283.

2. Kumar V, Abbas AK, Fausto N. Pathologic basis of disease. $7^{\text {th }}$ ed. Singapore: Elsevier.2007: 607-609.
3. Kenneth L. Special Report: Diagnosis of Myocarditis; Death of Dallas Criteria Circulation. 2006; 113: 593-595.

4. Aretz HT, Billingham ME, Edwards WD, Factor SM, Fallon JT, Fenoglio JJ Jr, Olsen EG, Schoen FJ. Myocarditis: a histopathologic definition and classification. American Journal of Cardiovascular Pathology. 1987; 1: 3-14.

5. Chow LH, Radio SJ, Sears TD, McManus BM. Insensitivity of right ventricular endomyocardial biopsy in the diagnosis of myocarditis. Journal of American College of Cardiology. 1989; 14: 915-920.

\section{CORRESPONDENCE}

Dr. R. H. A. I. Rathnaweera

Department of Forensic Medicine, University of Ruhuna, Sri Lanka.

E mail-ajithrathnaweera@gmail.com

Received: August 2017

Accepted: September 2017 